\title{
Crab dispersion and its impact on the CERN Large Hadron Collider collimation
}

\author{
Yi-Peng Sun (孙一鹏), ${ }^{*}$ Ralph Assmann, Rogelio Tomás, and Frank Zimmermann \\ European Organization for Nuclear Research (CERN), Geneva, Switzerland
}

(Received 19 October 2009; published 12 March 2010)

\begin{abstract}
Crab cavities are proposed to be used for a luminosity upgrade of the Large Hadron Collider (LHC). Crab cavities are rf cavities operated in a transverse dipole mode, which imparts on the beam particles a transverse kick that varies with the longitudinal position along the bunch. The crab cavity introduces another kind of dispersion to the particles which is $z$ dependent, and thus could complicate the beam dynamics and have an impact on the LHC collimation system. As for LHC, the off-momentum beta-beat and dispersion-beat already compromise the performance of the collimation system; the crab dispersion introduced by global crab cavities might do the same, and should be carefully evaluated. In this paper, we present a definition for the crab dispersion, and study its impact on the LHC collimation system.
\end{abstract}

\section{INTRODUCTION}

The design luminosity of the Large Hadron Collider (LHC) is $10^{34} \mathrm{~cm}^{-2} \mathrm{~s}^{-1}$ at the two high-luminosity proton-proton experiments ATLAS (located at interaction point 1, or IP1) and CMS (located at IP5), with a center-ofmass energy of $14 \mathrm{TeV}$ [1]. To further increase the LHC luminosity, several upgrade scenarios have crystallized from various considerations. For two of them, namely the early-separation scheme and the full crab crossing (FCC) scheme, crab cavities are an essential ingredient of the upgrade.

Crab cavities (CC) have been proposed for both linear [2] and circular colliders [3], to restore an effective headon collision at the IP. The crab cavity gives rise to a $z$-dependent transverse kick on the beam particles, as well as to a change in the longitudinal momentum. In standard accelerator coordinates, the required horizontal kick from the crab cavity can be written as (assuming horizontal beam-beam crossing)

$$
\Delta p_{x}=-\frac{\partial H_{\mathrm{crab}}}{\partial x}=-\frac{q V}{E_{s}} \sin \left(\phi_{s}+\frac{\omega z}{c}\right),
$$

where $\Delta p_{x}$ denotes the horizontal kick.

The Hamiltonian to describe the thin crab cavity is obtained by integration with respect to $x$ (in the Lie algebra sense $[4,5])$ :

$$
H_{\text {crab }}=\frac{q V}{E_{s}} \sin \left(\phi_{s}+\frac{\omega z}{c}\right) x,
$$

where $H_{\text {crab }}$ denotes the Hamiltonian, $q$ the particle charge, $V$ the voltage of the crab cavity, $E_{s}$ the particle energy, $\phi_{s}$ the synchronous phase of the crab-cavity rf wave, $\omega$ the angular frequency of the crab cavity, $z$ the longitudinal coordinate of the particle with respect to the bunch center, $c$ the velocity of light, and $x$ the horizontal coordinate.

\footnotetext{
*Yipeng.SUN@cern.ch
}

Because of symplecticity, the crab cavity also introduces an $x$-dependent longitudinal kick,

$$
\Delta p_{z}=-\frac{\partial H_{\mathrm{crab}}}{\partial z}=-\frac{q V}{E_{s}} \cos \left(\phi_{s}+\frac{\omega z}{c}\right) \frac{\omega}{c} x
$$

\section{CRAB DISPERSION}

The concept of $z$-dependent dispersion has been introduced by Ohmi and Forest et al. Similar more general dispersion functions have been described elsewhere, such as [6], but here we give explicit expressions and apply to the case of a crab cavity.

\section{A. Off-momentum dispersion}

For a particle with design momentum $P_{s}$, the ideal motion follows the central design orbit that goes through the center of all quadrupoles (assuming that there are no other errors). Now we consider a particle with the relative momentum offset $\delta=\Delta p / P_{s}$. Its trajectory certainly differs from the on-momentum one. The off-momentum dispersion $D(s)$ can be defined by [7]

$$
x\left(s, \frac{\Delta p}{P_{s}}\right)=D(s) \frac{\Delta p}{P_{s}},
$$

where the radial distance $x\left(s, \Delta p / P_{s}\right)$ from the design orbit is proportional to the relative momentum offset $\delta=$ $\Delta p / P_{s}$. This off-momentum particle satisfies the inhomogeneous Hill equation for the horizontal motion,

$$
x^{\prime \prime}+K(s) x=\frac{1}{\rho} \frac{\Delta p}{P_{s}},
$$

where $\rho$ denotes the ring curvature.

The total deviation of the trajectory from the reference orbit can be written as [7]

$$
x(s, \delta)=x_{D}(s, \delta)+x_{\beta}(s),
$$

where $x(s)$ denotes the total deviation, $x_{D}(s)=$ $D(s) \Delta p / P_{s}$ the deviation of the off-momentum closed 
orbit with a fixed $\Delta p$, and $x_{\beta}(s)$ the betatron oscillation around this closed dispersive orbit.

The periodic dispersion $D(s)$ satisfies the differential equation

$$
D^{\prime \prime}+K(s) D=\frac{1}{\rho(s)},
$$

and can be obtained as [7]

$$
D(s)=\frac{\sqrt{\beta(s)}}{2 \sin \pi Q} \oint \frac{\sqrt{\beta(t)}}{\rho(t)} \cos [|\Phi(t)-\Phi(s)|-\pi Q] d t,
$$

where $Q$ denotes the betatron tune of the storage ring, $\beta(s)$ the beta function at location $s$, and $\beta(t)$ the beta function at other locations.

\section{B. Crab dispersion for sinusoidal crab kick}

From the point of view of the closed orbit, the crab cavity's effect is similar to that of an orbit corrector, or the field error of a dipole. The difference is that the transverse kick is $z$ dependent, not momentum dependent. So we follow the approach of the closed-orbit distortion, to get the deviation of the crab closed orbit with a specified longitudinal position along the bunch

$$
x_{D_{c c}}(z, s)=\frac{\sqrt{\beta(s) \beta_{\mathrm{crab}}}}{2 \sin (\pi Q)} \Delta p_{1}(z) \cos \left(\Delta \varphi_{1}-\pi Q\right),
$$

where $x_{D_{c c}}(z)$ denotes the deviation of the crab closed orbit, $\beta(s)$ the beta function at the location $s, \beta_{\text {crab }}$ the beta function at the crab-cavity location, $Q$ the betatron tune of the storage ring, $\Delta p_{1}$ the deflecting angle for a particle at a specified longitudinal location $z$ (within the bunch), and $\Delta \varphi_{1}$ the phase advance (absolute value) between the crab-cavity location and the location $s$.

The total deviation of the trajectory from the reference orbit can be rewritten as

$$
x(s, z, \delta)=x_{D}(s, \delta)+x_{\beta}(s)+x_{D_{c c}}(z, s) .
$$

The effect of the crab cavity can be modeled by a horizontal dipole corrector (with $z$-dependent strength), with the deflecting angle as

$$
\Delta p_{1}(z)=-\frac{q V}{P_{s}} \sin \left(\frac{\omega z}{c}\right),
$$

where $\Delta p_{1}(z)$ denotes the kicking angle of the corrector, $q$ the particle charge, $P_{s}$ the longitudinal particle momentum, and $z$ the longitudinal coordinate of the particle with respect to the bunch center.

The required crab-cavity voltage (global crab cavity, or $\mathrm{GCC}$ ) for recovering the crossing angle $\theta$ at the interaction point (IP) is [5]

$$
V=\frac{c^{2} P_{s} \tan \left(\frac{\theta}{2}\right)}{q \omega \sqrt{\beta^{*} \beta_{\text {crab }}}}\left|\frac{2 \sin (\pi Q)}{\cos \left(\Delta \varphi_{0}-\pi Q\right)}\right|,
$$

where $V$ denotes the voltage of the global crab cavity, $c$ the velocity of light, $P_{s}$ the particle momentum, $\theta$ the full crossing angle, $\omega$ the angular frequency of the crab cavity, $\beta^{*}$ the beta function at the interaction point, and $\Delta \varphi_{0}$ the phase advance between the crab-cavity location and the IP.

Inserting formulas (11) and (12) into formula (9), we can get the deviation of the closed orbit from the $z$-dependent crab dispersion as

$$
x_{D_{c c}}(z, s)=\sqrt{\frac{\beta(s)}{\beta^{*}}} \frac{c \tan \left(\frac{\theta}{2}\right)}{\omega} \sin \left(\frac{\omega z}{c}\right) \frac{\cos \left(\Delta \varphi_{1}-\pi Q\right)}{\cos \left(\Delta \varphi_{0}-\pi Q\right)},
$$

where $\theta / 2$ denotes the half crossing angle (crabbing angle for each beam), $\omega$ the angular frequency of the crab cavity, $z$ the longitudinal coordinate of the particle with respect to the bunch center, $\Delta \varphi_{1}$ the phase advance between the crabcavity location and the location $s$, and $\Delta \varphi_{0}$ the phase advance between the crab-cavity location and the IP.

From formula (13), we observe that the deviation of the closed orbit from the $z$-dependent crab dispersion is proportional to the crabbing angle $\theta / 2$ (small angle case), and that it is modulated by the angular frequency of the crab cavity and the longitudinal coordinate $z$, as $\sin (\omega z / c) / \omega$.

To have a similar unit as for the off-momentum dispersion, for the case $\frac{c}{\omega} \gg \sigma_{z}$ (the crab kick is linear within $\left.\pm 1 \sigma_{z}\right)$, we define the crab dispersion as the deviation normalized by $1 \sigma_{p}$ with $z=1 \sigma_{z}$ :

$$
D_{c c}(s)=\frac{x_{D_{c c}}\left(1 \sigma_{z}, s\right)}{1 \sigma_{p}} .
$$

In an earlier report [8], we already summarized the possible installation and impact on the beam of a single global crab cavity for the nominal LHC optics and for one LHC upgrade optics ("lowbetamax" [9]). Here we consider the same nominal LHC optics and a global crabcavity scheme. The global crab cavity is installed at the reserved 200-MHz rf location for beam 1. The other parameters of the nominal LHC optics and lowbetamax optics are the same as listed in Ref. [10]. For LHC beam 1 and the nominal LHC collision optics, the corresponding parameters at IP5 and at the single global crab-cavity location are listed in Table I. Other parameters of the nominal LHC collision optics comprise the half crossing angle $\theta / 2=$ $142 \mu \mathrm{rad}, \quad \beta^{*}=0.55 \mathrm{~m}, \quad \sigma_{p}=0.00011, \quad$ and $\sigma_{z}=$ $0.075 \mathrm{~m}$.

With the nominal LHC optics and global crab-cavity scheme mentioned above, by using formulas (13) and (14), we get the crab dispersion for two crab frequencies $(800$ and $400 \mathrm{MHz}$ ), as shown in Fig. 1. For our definition (14), since at $1 \sigma_{z}$ and for LHC parameters there is already a marked difference from a linear rf slope, the crab dispersion is comparable with the off-momentum dispersion, and obviously the $400-\mathrm{MHz}$ case has a larger crab dispersion. At the specified IP (IP5), we get the required crab disper- 
TABLE I. Parameters at IP1\&IP5 and at the crab-cavity location (nominal LHC).

\begin{tabular}{|c|c|c|c|c|c|}
\hline & $\begin{array}{c}s \\
{[\mathrm{~m}]}\end{array}$ & $\begin{array}{c}\beta_{x} \\
{[\mathrm{~m}]}\end{array}$ & $\begin{array}{c}\beta_{y} \\
{[\mathrm{~m}]}\end{array}$ & $\begin{array}{c}\text { Phase } \\
\text { advance } \\
x(2 \pi)\end{array}$ & $\begin{array}{c}\text { Phase } \\
\text { advance } \\
y(2 \pi)\end{array}$ \\
\hline IP1 & 0 & 0.55 & 0.55 & 0 & 0 \\
\hline Crab cavity & 9968 & 208 & 174 & 24.38 & 21.84 \\
\hline IP5 & 13329 & 0.55 & 0.55 & 32.05 & 29.61 \\
\hline
\end{tabular}

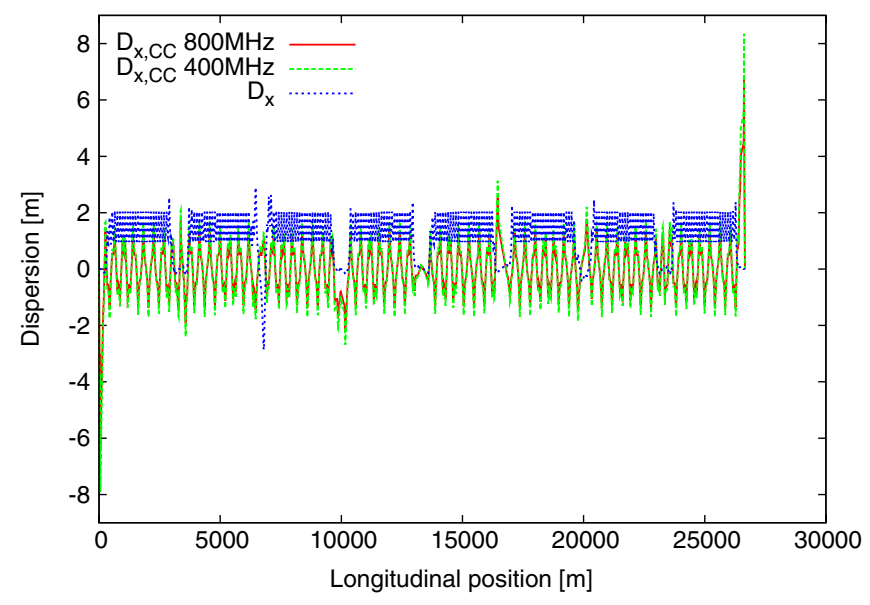

FIG. 1. (Color) Crab dispersion and off-momentum dispersion for nominal LHC optics.

sion to achieve the quasi-head-on collision. The crab dispersion has its peak in the triplet quadrupoles at both sides of IP5, as the $\beta(s)$ function is the largest there and also due to the phase advance $\Delta \varphi_{1}$. In the case of the lowbetamax collision optics for an upgrade LHC interaction region $[8,9]$, we have the half crossing angle $\theta / 2=200 \mu \mathrm{rad}$ and $\beta^{*}=0.25 \mathrm{~m}$. Here the crab dispersion is much larger, as shown in Fig. 2.

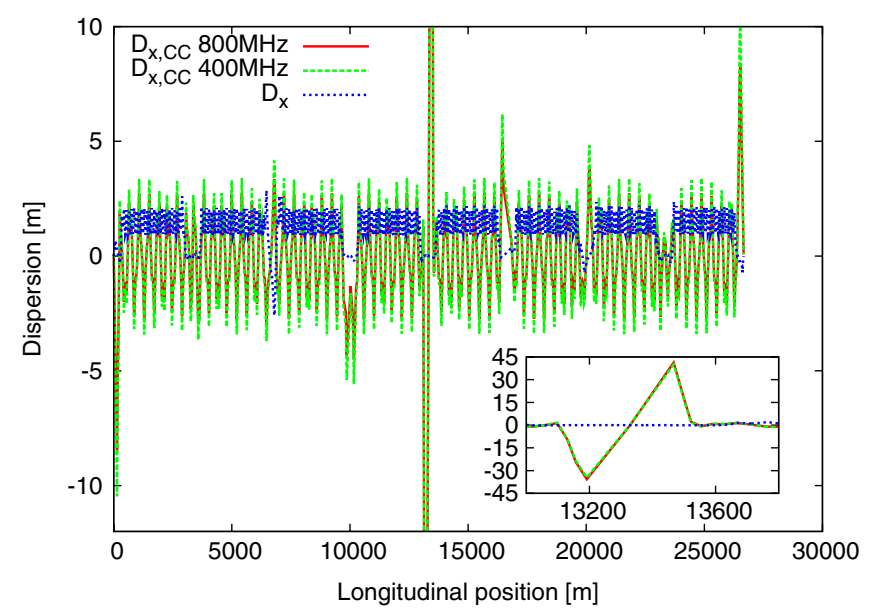

FIG. 2. (Color) Crab dispersion and off-momentum dispersion for lowbetamax collision optics.

\section{Crab dispersion for linear crab kick}

For the linear crab kick case, we have $\omega \Rightarrow 0$, so

$$
\frac{\sin (\omega z / c)}{\omega} \approx \frac{\omega z}{c \omega}=\frac{z}{c} .
$$

The orbit deviation from the $z$-dependent crab dispersion [formula (13)] simplifies to

$$
x_{D_{c c}}(z, s)=\sqrt{\frac{\beta(s)}{\beta^{*}}} \tan \left(\frac{\theta}{2}\right) z \frac{\cos \left(\Delta \varphi_{1}-\pi Q\right)}{\cos \left(\Delta \varphi_{0}-\pi Q\right)} .
$$

We observe that now $x_{D_{c c}}(z, s)$ is linearly proportional to $z$. So the crab dispersion can be defined as the deviation normalized by any $n \sigma_{p}$ with $z=n \sigma_{z}$ ( $n$ is a number not equal to zero). In this case, the crab dispersion can be written as

$$
D_{c c}(s)=\sqrt{\frac{\beta(s)}{\beta^{*}}} \tan \left(\frac{\theta}{2}\right) \frac{\sigma_{z}}{\sigma_{p}} \frac{\cos \left(\Delta \varphi_{1}-\pi Q\right)}{\cos \left(\Delta \varphi_{0}-\pi Q\right)} .
$$

At LHC IP5 where the beam 1 should be crabbed by $\theta / 2$, we have $\beta(s)=\beta^{*}$ and $\Delta \varphi_{0}-\pi Q=\Delta \varphi_{1}-\pi Q \approx$ $2 \pi$. The orbit deviation can then be further simplified to

$$
x_{D_{c c}, \mathrm{IP} 5}(z)=\tan \left(\frac{\theta}{2}\right) z .
$$

The result is plotted in Fig. 3, for crossing angles equal to 285 and $400 \mu \mathrm{rad}$, respectively, which shows the required $x-z$ correlation at IP.

\section{Comparison with simulation}

We compare the crab dispersive orbit from the theoretical prediction with the LHC simulations, by using formulas (2), (11), and (13), as well as the design TWISS parameters of the LHC at top energy mentioned above. For the simulation or in the real machine study, to measure the crab

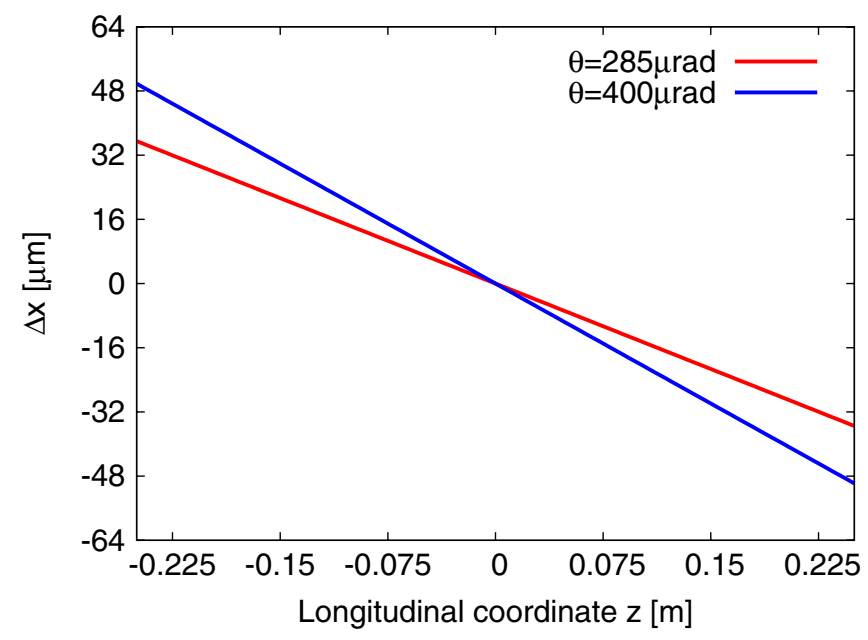

FIG. 3. (Color) The orbit deviation at IP5 with a linear crab kick (ideally low crab frequency), for two different crossing angles. 


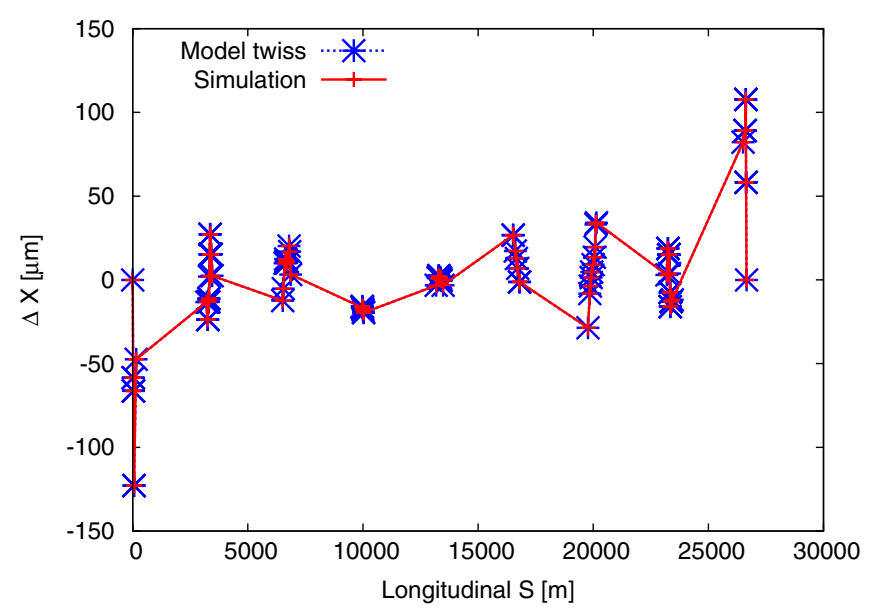

FIG. 4. (Color) Comparison of crab dispersive orbit between theoretical results (blue) and LHC simulation (red).

orbit of the bunch by beam position monitor, the rf phase of the $800-\mathrm{MHz}$ global crab cavity is shifted by $10^{\circ}$ artificially to create the crab dispersive orbit. A good agreement is found between the theoretical prediction and the simulation, as shown in Fig. 4.

\section{IMPACT ON LHC COLLIMATION}

Considering the huge beam power of LHC and to prevent even tiny loss of beam particles on cold surfaces, the LHC collimation system [11-13] is designed to absorb the beam halo outside of a specified transverse amplitude. Given the condition that the secondary collimators should never be hit by the primary beam halo, the closed orbit shall be strictly controlled in order to set the right jaw opening of collimators and to preserve the collimator hierarchy. As the global crab cavity introduces the $z$-dependent crab dispersion, it is essential to study the impact on the LHC collimation performance. In this paper we present the results for the nominal LHC collision optics, considering an initially on-momentum betatron beam halo, and with the above-mentioned $800-\mathrm{MHz}$ global crab cavity.

To generate a realistic $1 \mu \mathrm{m}$ impact parameter on the primary collimator with $6 \sigma$ half gap, for the normal LHC collimation simulation at top energy, the horizontal onmomentum beam halo is generated at $5.958 \sigma$ with
$0.0015 \sigma$ as smear. The so-called phase-one collimators are used without magnet errors, and the collimator jaw center is set to be on the actual closed orbit. In total, 5760000 macroparticles are generated as beam halo and tracked for 200 turns.

To investigate the impact of the $z$-dependent crab dispersion on the collimation cleaning inefficiency, we treat the global crab cavity as a dipole corrector (with the strength as the crab kick at different longitudinal offset, such as $z=1 \sigma_{z}, z=2 \sigma_{z} \ldots$ ), and set the collimator jaw center with respect to the center of the vacuum chamber. This convention is achieved by modifying the collimation part in the simulation code SIXTRACK [14]. By this trick we can study the impact of the crab dispersion for different longitudinal slices along the bunch, and the synchrotron oscillation is effectively frozen during the collimation tracking. Based on the crab rf frequency $800 \mathrm{MHz}$, and the LHC $\mathrm{rf}$ bucket size, four cases $\left(z=1 \sigma_{z}, z=2 \sigma_{z}, z=\right.$ $3 \sigma_{z}$, and $z=4 \sigma_{z}$ ) are selected for the collimation simulations with a global crab cavity.

As these four cases have different crab dispersion $D_{c c}(z, s)$, the closed orbit is also different. To maintain a $1 \mu \mathrm{m}$ impact parameter for the first turn, the initial beam halo is generated based on the change of the horizontal orbit $\Delta x$ at the primary collimator "TCP.C6L7.B1." The change of the horizontal orbit at the primary collimator "TCP.C6L7.B1," the initial halo position and smear, the average first turn and all-turns impact parameter, and the particles absorbed within 200 turns are summarized in Table II, for these four cases.

\section{A. Phase space cut}

\section{Impact of off-momentum beat}

The impact of off-momentum beta-beat (dispersionbeat) on the effective LHC collimator settings is studied, for the nominal LHC collision optics (with beta-beat corrected in one-half of the LHC ring) [11]. In LHC, the collimator jaw half gap is set to be $r_{\text {cut }}(r$ stands for transverse coordinates $x$ or $y$ ), and the jaws are ideally centered around the closed orbit. For the primary betatroncleaning collimators, usually we have $r_{\text {cut }}=6 \sigma$, and for the secondary betatron-cleaning collimators $r_{\text {cut }}=7 \sigma$.

For each collimator, the phase space cut on the beam by the nominal collimator settings can be defined as [11]

TABLE II. Collimation simulation parameters for different cases, with the 800-MHz global crab cavity.

\begin{tabular}{lcccc}
\hline \hline & $\mathrm{GCC}\left(1 \sigma_{z}\right)$ & $\mathrm{GCC}\left(2 \sigma_{z}\right)$ & $\mathrm{GCC}\left(3 \sigma_{z}\right)$ & $\mathrm{GCC}\left(4 \sigma_{z}\right)$ \\
\hline$\Delta x @$ primary collimator "TCP.C6L7.B1" $[\sigma]$ & -0.470 & -0.285 & 0.300 & 0.466 \\
Initial halo $[\sigma]$ & 5.509 & 5.668 & 5.662 & 5.500 \\
Initial smear $[\sigma]$ & 0.0015 & 0.0015 & 0.0015 & 0.0015 \\
Impact parameter, 1st turn $[\mu \mathrm{m}]$ & 1.3 & 1.5 & 1.0 & 0.95 \\
Impact parameter, all turns $[\mu \mathrm{m}]$ & 14.0 & 14.9 & 14.7 & 13.8 \\
Particle absorbed & $69.1 \%$ & $69.0 \%$ & $68.4 \%$ & $68.1 \%$ \\
\hline \hline
\end{tabular}




$$
r_{\mathrm{cut}}\left(i_{\mathrm{coll}}\right)=n_{\beta, \mathrm{cut}}\left(i_{\mathrm{coll}}, \delta\right) \sqrt{\epsilon_{r} \beta_{r}\left(i_{\mathrm{coll}}, \delta\right)}+D_{r}\left(i_{\mathrm{coll}}, \delta\right) \delta,
$$

where $r_{\text {cut }}\left(i_{\text {coll }}\right)$ denotes the half gap of the collimator jaw at the $i$ th collimator, $n_{\beta, \text { cut }}\left(i_{\text {coll }}, \delta\right)$ the effective betatron amplitude cut at the $i$ th collimator, $\delta=\Delta p / p$ the energy offset, $\epsilon_{r}$ the beam transverse emittance, $\beta_{r}\left(i_{\text {coll }}, \delta\right)$ the beta function at the $i$ th collimator, and $D_{r}\left(i_{\text {coll }}, \delta\right)$ the dispersion at the $i$ th collimator.

If we consider both collimator jaws and a sufficient time for longitudinal and horizontal phase space motion, the effective betatron amplitude cut at the $i$ th collimator can be written as [11]

$$
n_{\beta, \mathrm{cut}}\left(i_{\mathrm{coll}}, \delta\right)=\frac{ \pm r_{\mathrm{cut}}\left(i_{\mathrm{coll}}\right)-D_{r}\left(i_{\mathrm{coll}}, \delta\right) \delta}{\sqrt{\epsilon_{r} \beta_{r}\left(i_{\mathrm{coll}}, \delta\right)}} .
$$

In Fig. 5 such an effective betatron amplitude cut is shown for the primary betatron-cleaning collimator TCP.C6L7.B1 (with $D_{x}=0.38 \mathrm{~m}$ ). The red curve corresponds to the exact result of Eq. (20), which includes $\beta$ beating and higher-order dispersion. If the off-momentum beta-beat is not included, and we do not consider the dispersion $D_{r}\left(i_{\text {coll }}, \delta\right)$ at this collimator, we get the effective betatron amplitude cut as the blue dashed line in Fig. 5. For another case, if the off-momentum beta-beat and highorder dispersion are not included, but with the constant linear dispersion $D_{r}\left(i_{\text {coll }}\right)$ at this collimator in consideration, we get the effective betatron amplitude cut as the green dashed line in Fig. 5 (which is different from the blue dashed line and tilted by the dispersion). At last, with offmomentum beta-beat, the real phase space cut is shown as the red line in Fig. 5. In Fig. 6 a similar effective phase space cut is plotted for the primary momentum-cleaning collimator TCP.6L3.B1 (with $D_{x}=2.2 \mathrm{~m}$ ). The slope of the curve is determined by the dispersion at the specified collimator, and the tilt is modulated by the off-momentum beta-beat and dispersion-beat.

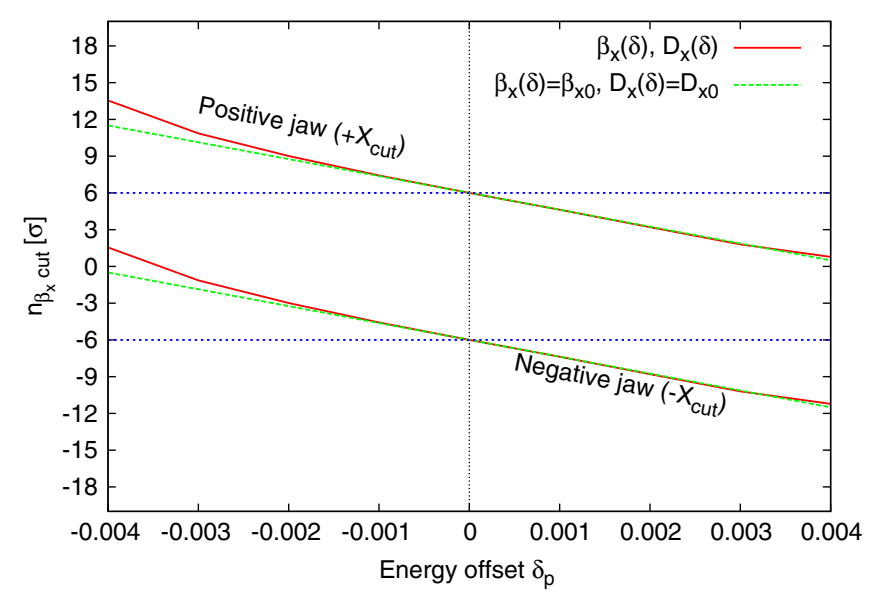

FIG. 5. (Color) Off-momentum phase space cut at the primary collimator TCP.C6L7.B1 (with $D_{x}=0.38 \mathrm{~m}$ ).

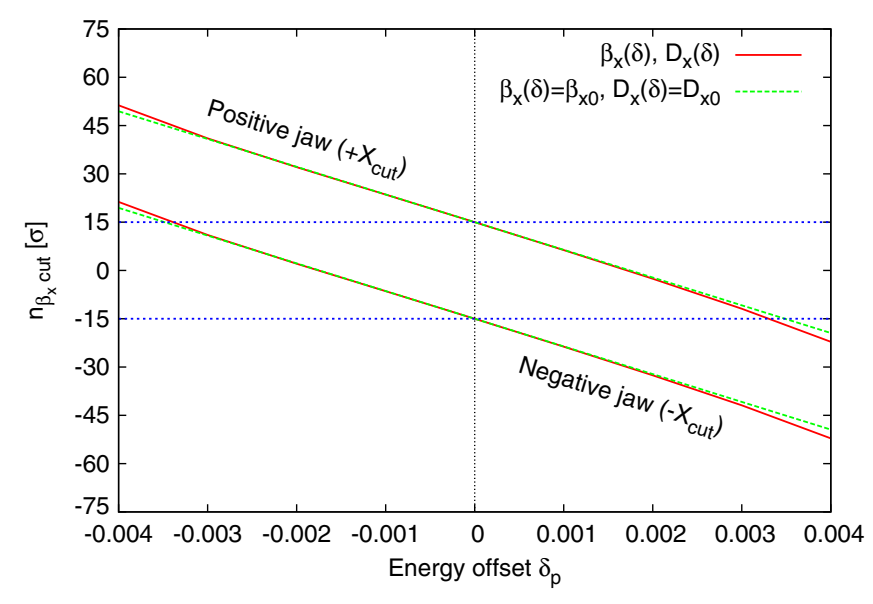

FIG. 6. (Color) Off-momentum phase space cut at the primary collimator TCP.6L3.B1 (with $D_{x}=2.2 \mathrm{~m}$ ).

There are two main regions where LHC collimators are concentrated: IR3 (momentum cleaning) and IR7 (betatron cleaning). The collimators are separated into different groups based on their functions, such as the primary collimators in IR3\&IR7 (TCP), the secondary collimators in IR3\&IR7 (TCSG), the tertiary collimators (TCTH), the absorbers for showers in cleaning insertions in IR3\&IR7 (TCLA), and the collimators to protect the machine during beam dump (TCDQ) etc. In Table III the half gaps of the different groups of LHC collimators are listed, where TCDQ is only in IR6 and TCTH is in IR1\&IR2\&IR5\&IR8. In Fig. 7 the phase space cut from purely off-momentum beat is shown together with the size of the rf bucket. The figure demonstrates the hierarchy of primary (TCP), secondary (TCSG), tertiary (TCTH), beam dump (TCDQ) horizontal collimators, and shower absorbers (TCLA), and confirms previous results [11]. The dashed lines represent the reflections of the calculated solid curves from formula (22) with respect to the vertical zero axis $\left[n_{\beta, \text { cut }}\left(i_{\text {coll }}, \delta\right)=0\right]$, which are imposed by phase space mixing (valid for a much slower synchrotron oscillation than betatron oscillation, and stable beam) [11].

\section{Impact of crab dispersion}

Now we look at formula (19) and add crab dispersion to it. Without crab dispersion, $r_{\text {cut }}\left(i_{\text {coll }}\right)$ is a function of

TABLE III. The half gap of the different group of LHC collimators (in unit of rms beam size).

\begin{tabular}{lcc}
\hline \hline & IR7 & IR3 \\
\hline TCP & 6 & 15 \\
TCSG & 7 & 18 \\
TCLA & 10 & 20 \\
TCTH (IR1\&IR2\&IR5\&IR8) & 8.3 & 8.3 \\
TCDQ (IR6) & 8 & 8 \\
\hline \hline
\end{tabular}




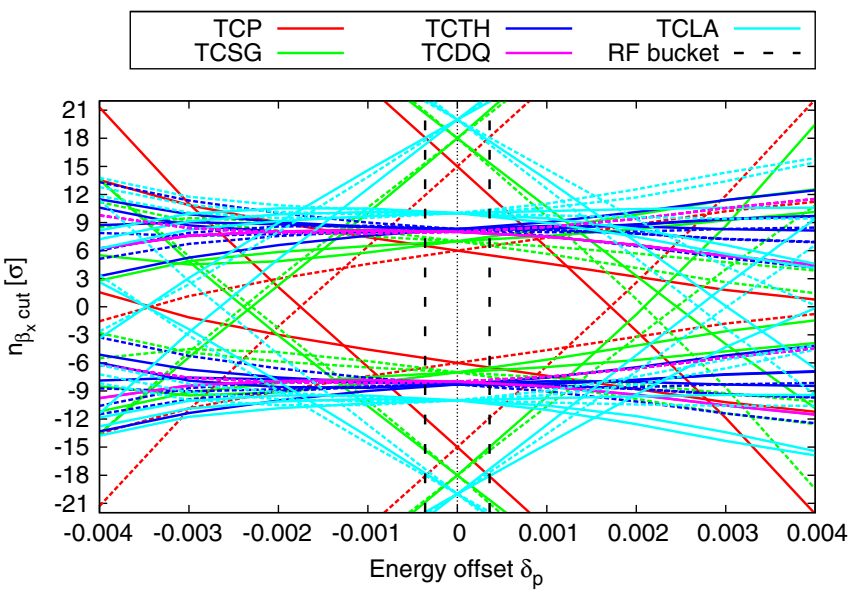

FIG. 7. (Color) Phase space cut from purely off-momentum beat, revealing the hierarchy of primary (TCP), secondary (TCSG), tertiary (TCTH), beam dump (TCDQ) horizontal collimators, and shower absorbers (TCLA).

$\beta_{r}\left(i_{\text {coll }}, \delta\right), D_{r}\left(i_{\text {coll }}, \delta\right)$, and $\delta$, so that $r_{\text {cut }}\left(i_{\text {coll }}\right)$ is a function of $\delta$. With crab dispersion, $r_{\text {cut }}\left(i_{\text {coll }}\right)$ is a function of both $\delta$ and $z$. We can rewrite formula (19) as

$$
\begin{aligned}
r_{\text {cut }}\left(i_{\text {coll }}\right)= & n_{\beta, \text { cut }}\left(i_{\text {coll }}, \delta\right) \sqrt{\epsilon_{r} \beta_{r}\left(i_{\text {coll }}, \delta\right)} \\
& +D_{r}\left(i_{\text {coll }}, \delta\right) \delta+x_{D_{c c}}(z, s),
\end{aligned}
$$

where $x_{D_{c c}}(z, s)$ denotes the deviation of the closed orbit due to the $z$-dependent crab dispersion, $z$ the longitudinal coordinate of the particle with respect to the bunch center, and $s$ the longitudinal location in the ring.

With crab dispersion included, the effective betatron amplitude cut at the $i$ th collimator can be written as

$$
\begin{aligned}
n_{\beta, \mathrm{cut}}\left(i_{\mathrm{coll}}, \delta, z\right)= & \frac{1}{\sqrt{\epsilon_{r} \beta_{r}\left(i_{\mathrm{coll}}, \delta\right)}}\left[ \pm r_{\mathrm{cut}}\left(i_{\mathrm{coll}}\right)\right. \\
& \left.-D_{r}\left(i_{\mathrm{coll}}, \delta\right) \delta-x_{D_{c c}}(z, s)\right] .
\end{aligned}
$$

At the primary collimator TCP.6L3.B1, we have offmomentum dispersion $D_{x}=2.21 \mathrm{~m}$ and crab dispersion $D_{c c}\left(1 \sigma_{z}\right)=-1.1 \mathrm{~m}$. We take the most pessimistic case $D_{c c}\left(1 \sigma_{z}\right)=-1.1 \mathrm{~m}\left(\right.$ as $\left.\left|D_{c c}(z)\right| \leq 1.1 \mathrm{~m}\right)$, and then to get a first idea of the effect, we use formula (22) to calculate the phase space cut only with crab dispersion [neglecting the off-momentum dispersion $D_{r}\left(i_{\text {coll }}, \delta\right)$ ] for the primary collimator TCP.6L3.B1, as shown in Fig. 8. The curve is modulated by the crab dispersion $D_{c c}(z)$ at this specified collimator. The crab rf frequency $800 \mathrm{MHz}$ is reflected in the modulation period.

Considering the $z$-dependent crab dispersion and $z$-dependent betatron amplitude cut [formula (22)], and neglecting the off-momentum dispersion $D_{r}\left(i_{\text {coll }}, \delta\right)$, the available phase space from all the horizontal collimators

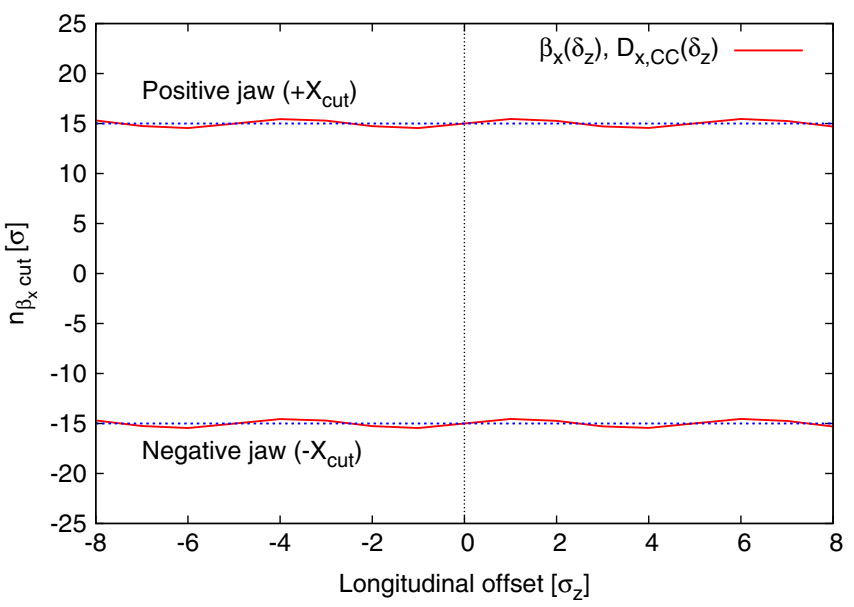

FIG. 8. (Color) Phase space cut with crab dispersion from the primary collimator TCP.6L3.B1.

[primary (TCP), secondary (TCSG), tertiary (TCTH), beam dump (TCDQ) collimators, and shower absorbers (TCLA)] can be calculated. Figure 9 shows the result. We observe that the crab dispersion's impact on the phase space cut is small. The maximum change on the phase space cut is $0.5 \sigma$, for the crab dispersion $D_{c c}(z) \approx$ $D_{c c}\left( \pm 1 \sigma_{z}\right), D_{c c}\left( \pm 4 \sigma_{z}\right)$, etc.

In Fig. 9 which is the available phase space from all the horizontal collimators (for $n_{\beta \text {,cut }}$ between $5 \sigma$ and $11 \sigma$ ) together with an indication of the nominal LHC rf bucket, we clearly observe this $0.5 \sigma$ perturbation. Also we notice that at the most pessimistic points $\left[D_{c c}(z)=D_{c c}\left( \pm 1 \sigma_{z}\right)\right]$ the primary and secondary collimators still keep their respective roles and the hierarchy is maintained.

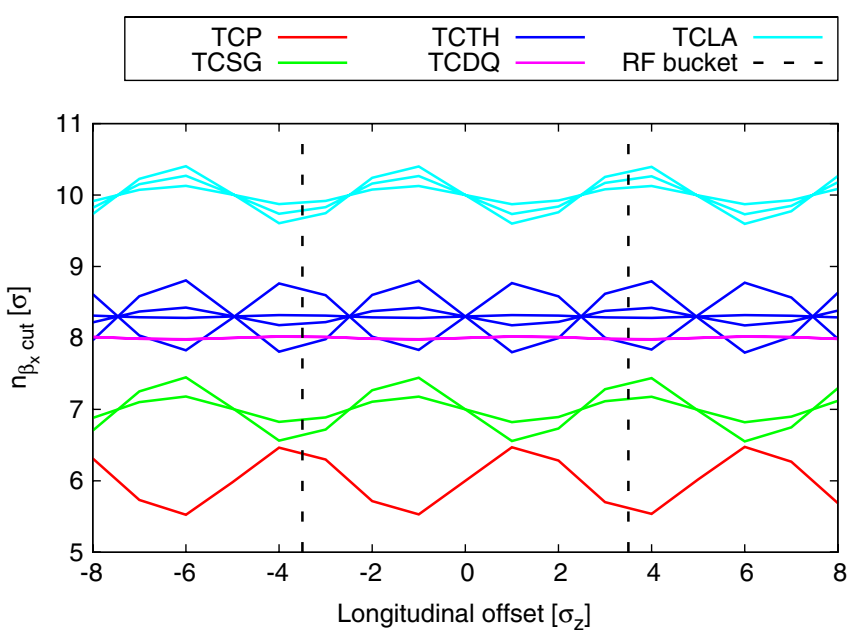

FIG. 9. (Color) Phase space cut from crab dispersion (for $n_{\beta \text {,cut }}$ between $5 \sigma$ and $11 \sigma$ ), with the hierarchy of primary (TCP), secondary (TCSG), tertiary (TCTH), beam dump (TCDQ) horizontal collimators, and shower absorbers (TCLA). 


\section{General case}

To combine the effects of off-momentum dispersion and crab dispersion, we can introduce a general longitudinal amplitude,

$$
A_{z}=\sqrt{\delta_{p}^{2}+\tilde{\delta}_{z}^{2}}
$$

where $A_{z}$ denotes the general amplitude, $\delta_{p}$ the energy offset, and $\tilde{\delta}_{z}$ the normalized crab offset from the crab cavity.

We also introduce a function $\Delta r\left(\delta_{p}, \tilde{\delta}_{z}\right)$ to describe the total orbit deviation from off-momentum dispersion and crab dispersion, as

$$
\Delta r\left(\delta_{p}, \tilde{\delta}_{z}\right)=D_{r}(s) \delta_{p}+D_{c c}(z, s) \tilde{\delta}_{z}
$$

where $D_{r}(s)$ denotes the dispersion at longitudinal location $s, D_{c c}(z, s)$ the crab dispersion at longitudinal location $s$ and for a particle with distance $z$ from the bunch center.

Theoretically, for any given $D_{r}(s)$ and $D_{c c}(z, s)$, and for a fixed total amplitude $A_{z}, \Delta r\left(\delta_{p}, \tilde{\delta}_{z}\right)$ will have a maximum (or minimum) value and the corresponding values of $\delta_{p}$ and $\tilde{\delta}_{z}$ can be determined.

We take the collimator TCP.C6L7.B1 as an example, where $D_{x}=0.38 \mathrm{~m}$ and $D_{c c}\left(1 \sigma_{z}\right)=-1.2 \mathrm{~m}$. At this collimator, we can solve Eqs. (23) and (24) with a fixed total amplitude $A_{z}=1 \sigma_{p}=1.1 \times 10^{-4}$, and find the solution $\delta_{p}=0.302 \sqrt{A_{z}}$. A schematic is shown in Fig. 10.

As $D_{r}(s)$ and $D_{c c}(z, s)$ are different at each collimator, and also due to the reason that $D_{c c}(z, s)$ has a dependence on $z$ which is nonlinear outside $\pm 1 \sigma_{z}$, it is difficult to solve $D_{r}(s)$ and $D_{c c}(z, s)$ for every given total amplitude $A_{z}$ (even inside the rf bucket) and all collimators. Here as the energy offset $\delta_{p}$ we consider is in the range of $-4 \times$

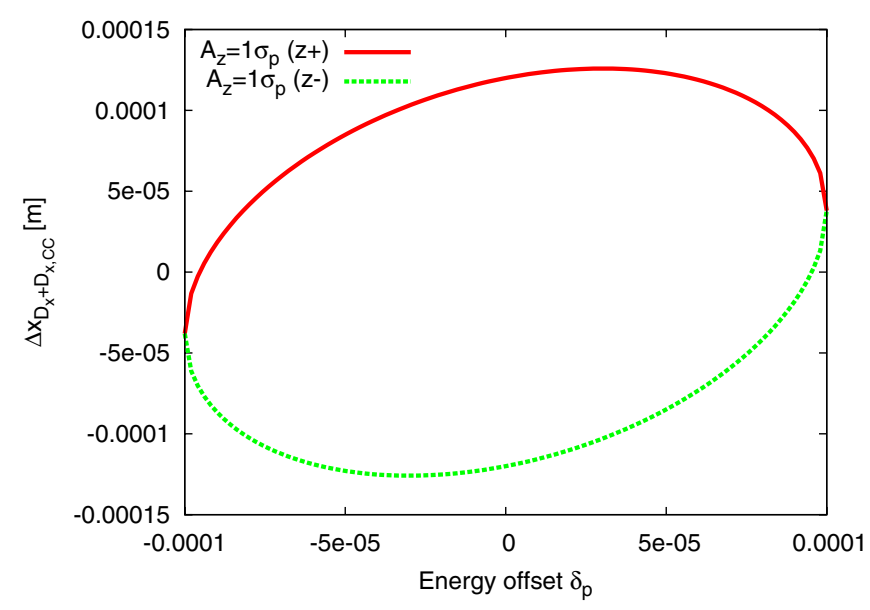

FIG. 10. (Color) Total orbit deviation versus energy offset $\delta_{p}$, at the primary collimator TCP.C6L7.B1, with both off-momentum dispersion and crab dispersion.
$10^{-4}$ and $4 \times 10^{-4}$, the contribution from the higher-order terms in the dispersion $D_{r}(s)$ is insignificant and can be neglected.

Again, for simplicity, we consider several cases along the bunch (within several fixed longitudinal slices) corresponding to $D_{c c}(z)=D_{c c}\left(1 \sigma_{z}\right)$ [or $D_{c c}(z)=D_{c c}\left(2 \sigma_{z}\right)$, $\left.D_{c c}(z)=D_{c c}\left(3 \sigma_{z}\right), D_{c c}(z)=D_{c c}\left(4 \sigma_{z}\right)\right]$ at each collimator, and then compute the phase space cut disturbed by the crab cavity (together with the off-momentum dispersion) by using formula (22). The result is shown in Fig. 11. We observe that the available beam phase space is similar to the one shown in Fig. 7, but modulated by the crab dispersion, while the collimator hierarchy is always maintained.

Figure 12 shows a zoomed view of Fig. 11 (top left), for the case considering the longitudinal slice with $z=1 \sigma_{z}$. As illustrated by formula (22), with some specified energy offset $\delta_{p}$, the deviation of the closed orbit due to the $z$-dependent crab dispersion and from the off-momentum dispersion can cancel each other [when $D_{r}\left(i_{\text {coll }}, \delta\right) \delta+$ $x_{D_{c c}}(z, s)=0$ ], which is denoted by the dots in Fig. 12 (for the primary and secondary collimators, TCP and TCSG).

As already stated, the collimator hierarchy has to be preserved and a proper margin has to be maintained in phase space between different groups of LHC collimators. For example, the design phase space distance between the primary (TCP) and secondary (TCSG) betatron-cleaning collimators is $1 \sigma$. For other groups the design distance can be inferred from Table III. The crab dispersion's impact on the minimum phase space distance is evaluated along the bunch between different groups of collimators, with results shown in Fig. 13. We observe that the minimum distance between the primary (TCP) and secondary (TCSG) betatron-cleaning collimators is $0.72 \sigma$; the minimum distance between the secondary (TCSG) and tertiary (TCTH) betatron-cleaning collimators is $0.92 \sigma$; the distance between the secondary (TCSG) betatron-cleaning collimators and the shower absorbers (TCLA) is almost preserved at $2 \sigma$; and the distance between the secondary (TCSG) betatron-cleaning collimators and the beam dump (TCDQ) collimators is increased to $1.19 \sigma$ at some longitudinal positions along the bunch.

\section{B. Global loss map}

The collimation simulations with the above-mentioned setups are performed for the nominal LHC collision optics (top energy, $\beta_{\mathrm{IP} 1,5}^{*}=0.55 \mathrm{~m}$ ), and the cleaning inefficiency is evaluated and compared between different cases. First we look at the global loss map. The cold magnets of LHC could be damaged by the halo particles that escape the collimators (primary, secondary, ....). So it is important to define and study the cleaning efficiency of the LHC collimation system. The so-called "global cleaning inefficiency" is defined as the leakage rate for a specified aperture $A_{c}[12]$, 

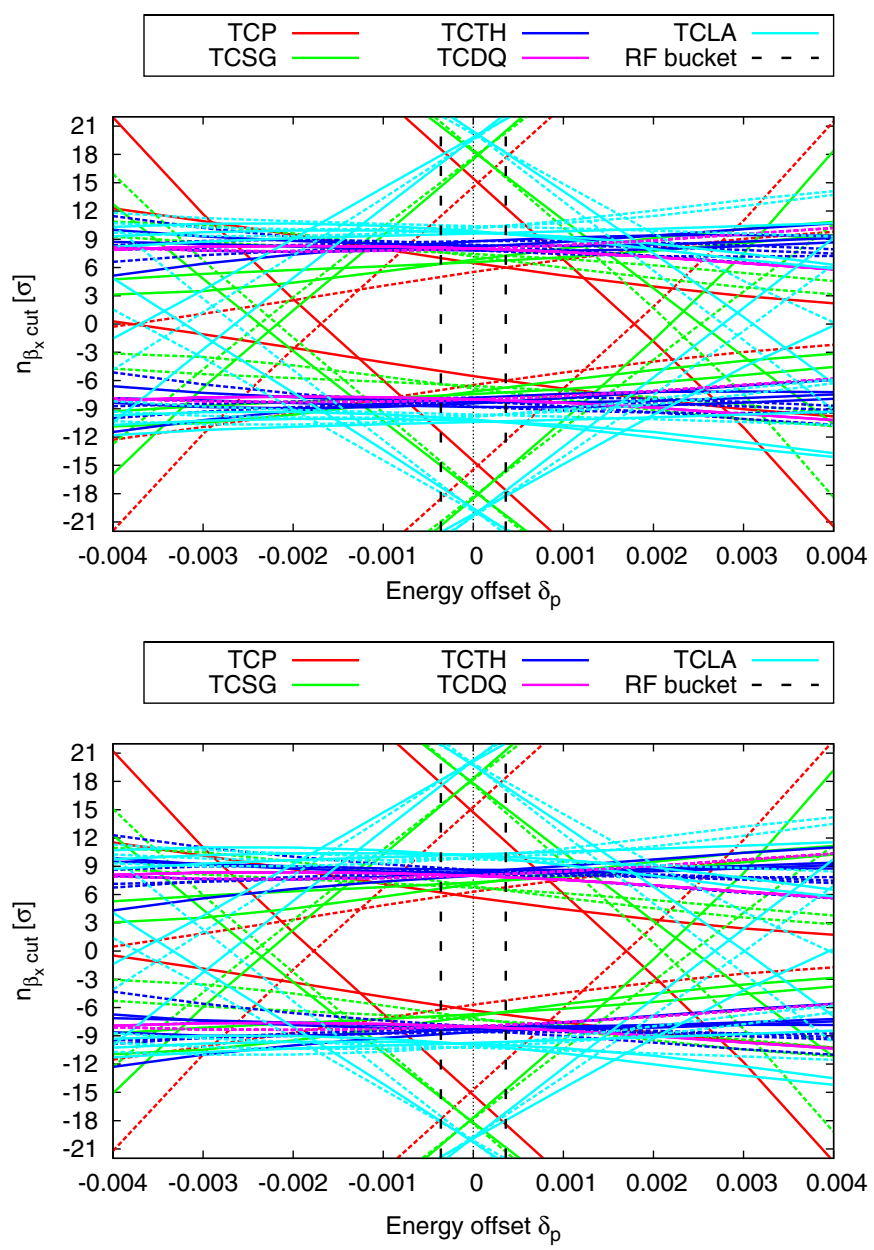

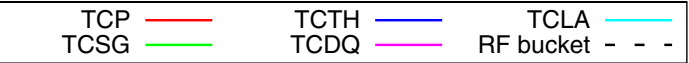
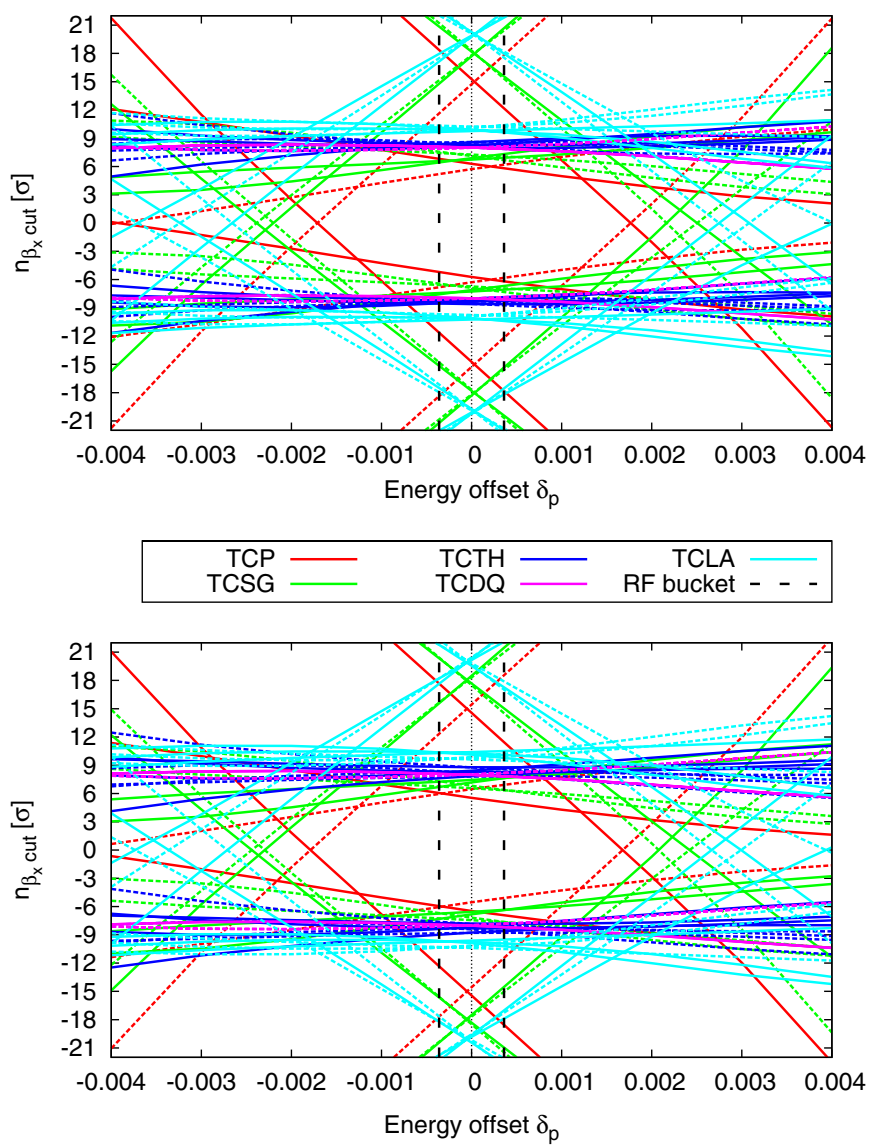

FIG. 11. (Color) CC disturbed phase space cut, with the hierarchy of primary (TCP), secondary (TCSG), tertiary (TCTH), beam dump (TCDQ) horizontal collimators, and shower absorbers (TCLA): longitudinal slice with $z=1 \sigma_{z}$ (top left); longitudinal slice with $z=2 \sigma_{z}$ (top right); longitudinal slice with $z=3 \sigma_{z}$ (bottom left); longitudinal slice with $z=4 \sigma_{z}$ (bottom right).

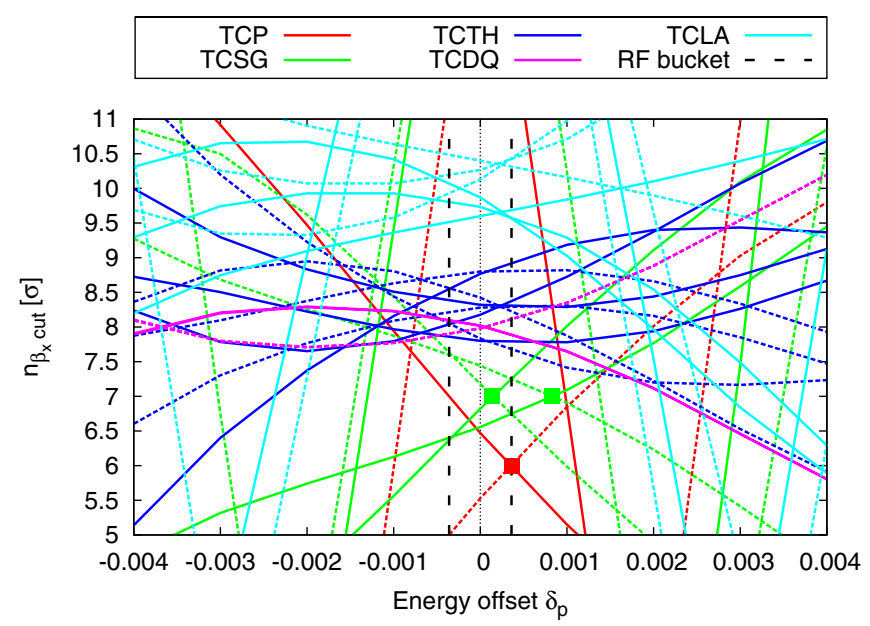

FIG. 12. (Color) Zoomed view of Fig. 11 (top left), longitudinal slice with $z=1 \sigma_{z}$.

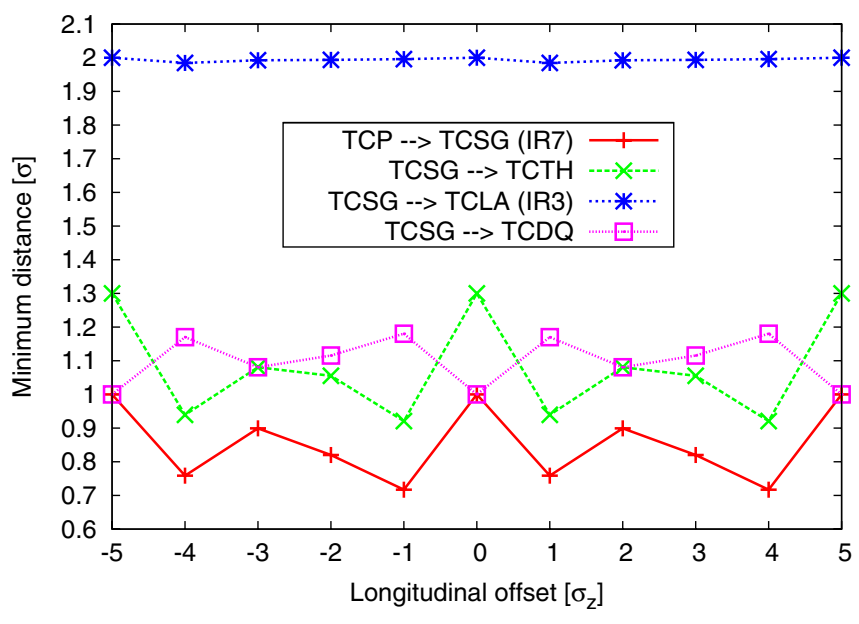

FIG. 13. (Color) Minimum phase space distance between different groups of collimators: TCP and TCSG (red); TCSG and TCTH (green); TCSG and TCLA (blue); TCSG and TCDQ (magenta). 


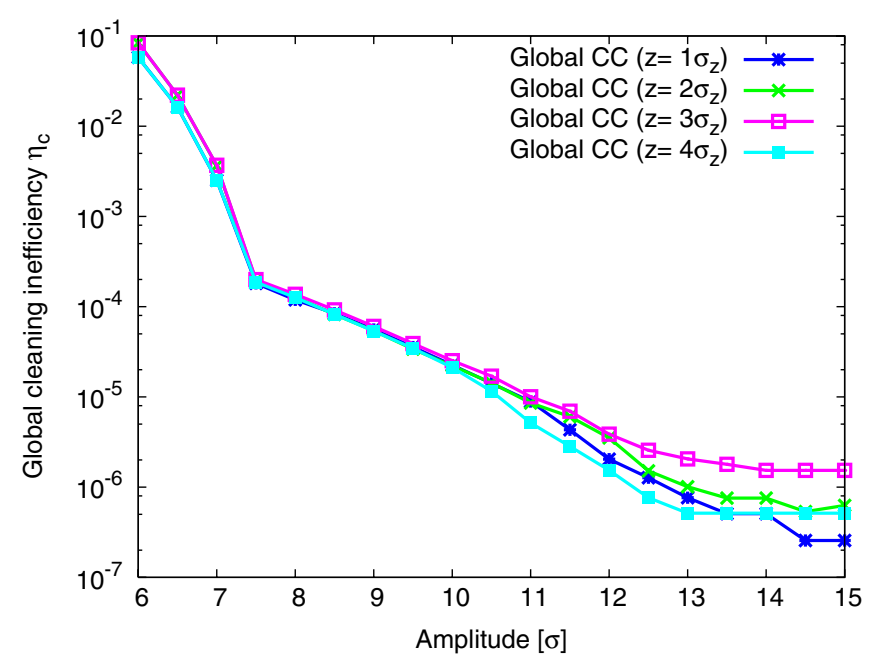

FIG. 14. (Color) Global cleaning inefficiency for LHC top energy, $\beta_{\mathrm{IP} 1,5}^{*}=0.55 \mathrm{~m}$, with the $800-\mathrm{MHz}$ global crab cavity: beam halo with $z=1 \sigma_{z}$ (blue); beam halo with $z=2 \sigma_{z}$ (green); beam halo with $z=3 \sigma_{z}$ (magenta); beam halo with $z=$ $4 \sigma_{z}$ (cyan).

$$
\eta_{c}\left(A_{c}\right)=\frac{N_{p}\left(A>A_{c}\right)}{N_{\mathrm{abs}}},
$$

where $N_{p}\left(A>A_{c}\right)$ denotes the number of particles which escape from the cleaning elements with a normalized amplitude $A>A_{c}$, and $N_{\text {abs }}$ is the total number of particles that undergo inelastic interactions with the collimator jaw.

The global cleaning inefficiency for different cases is shown in Fig. 14, which includes the results for the beam halo generated and tracked at the fixed longitudinal position $z=1 \sigma_{z}, z=2 \sigma_{z}, z=3 \sigma_{z}$, and $z=4 \sigma_{z}$, respectively. Only a small difference is found between these different cases which are all above the limit $\eta_{c}(10 \sigma)<$ $10^{-3}$. In particular, for all the cases we have $N_{p}(A>$ $12 \sigma)<30$, which is within the range of statistical error. Also the comparison is made between the global cleaning inefficiency in Fig. 14 and the case without crab cavity, and again only a small difference is found.

\section{Local loss map}

However, even if the global cleaning inefficiency $\eta_{c}$ (for $A_{c}=10 \sigma$ ) is under $10^{-3}$ which is the limit for the LHC at $7 \mathrm{TeV}$ top energy, it is still possible that the cold magnets could be quenched by the local concentration of the particle losses. In view of this consideration, it is much more important to study the distribution of the halo particles along the ring, and the so-called "local cleaning inefficiency" is defined as [12]

$$
\tilde{\eta}_{c}=\frac{\eta_{c}}{L_{\mathrm{dil}}},
$$

where $L_{\text {dil }}$ is the dilution length of the losses with a longitudinal resolution up to $10 \mathrm{~cm}$.

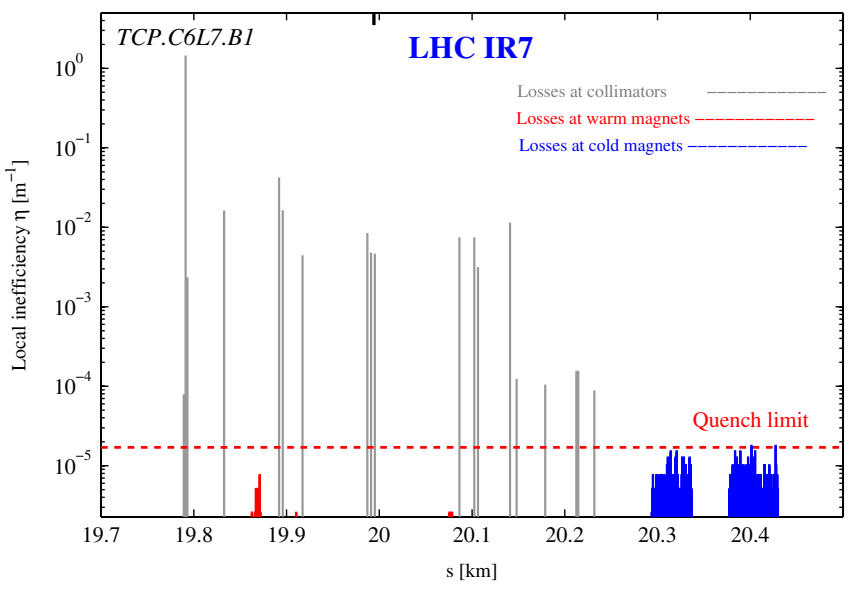

FIG. 15. (Color) Local cleaning inefficiency in LHC IR7, without global crab cavity (top energy, $\beta_{\mathrm{IP} 1,5}^{*}=0.55 \mathrm{~m}$ ).

A similar collimation simulation is performed for the nominal LHC collision optics, with and without the 800$\mathrm{MHz}$ global crab cavity, and with the above-mentioned convention. The local loss map for the case without the 800-MHz global crab cavity, as shown in Fig. 15, confirms with the previous result of the collimation team [11].

The local loss map in LHC IR7 for several different cases is shown in Fig. 16 (with the 800-MHz global crab cavity, and the beam halo is generated at four specified longitudinal positions along the bunch, namely $1 \sigma_{z}, 2 \sigma_{z}$, $3 \sigma_{z}$, and $4 \sigma_{z}$ ). We observe that most of the halo particles are absorbed by the primary collimator "TCP.C6L7.B1" which spark is indicated in the figure. Some halo particles are absorbed by the nearby vertical primary collimators after they are scattered by the horizontal primary collimator "TCP.C6L7.B1." The generated secondary beam halo is mostly absorbed by the secondary collimators downstream of the primary ones. The loss on the cold magnets is mainly in the dispersion suppressor section downstream of IP7, where the halo particles escaped from the collimator jaws are lost due to the large dispersion (also due to the energy change). Furthermore, if we compare the local loss maps of the different cases in Fig. 16 with the case without global crab cavity shown in Fig. 15, we find that they are similar and the cold losses are mostly still lower than the quench limit.

\section{CONCLUSION}

A crab dispersion function has been defined and its analytical expression derived. Its impact on the LHC collimation system has been studied. The study revealed a moderate degradation of the collimator hierarchy, which appears acceptable for global crab-cavity prototype tests in the LHC. In conclusion, the LHC collimation efficiency and collimator hierarchy appear insensitive to the presence of the global crab cavity for the nominal LHC optics. 

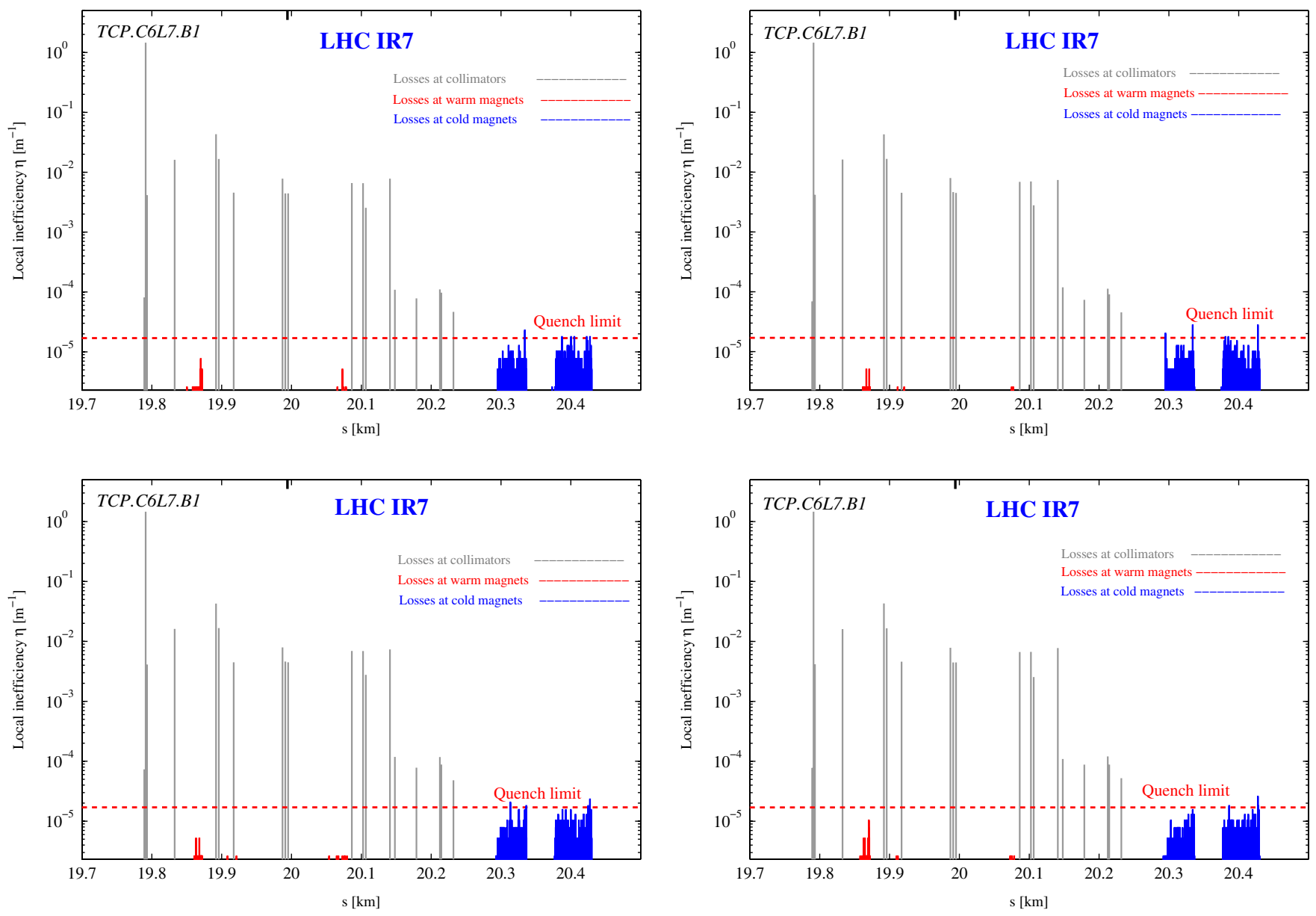

FIG. 16. (Color) Local cleaning inefficiency in LHC IR7, simulated with the $800-\mathrm{MHz}$ global crab cavity (top energy, $\beta_{\mathrm{IP} 1,5}^{*}=$ $0.55 \mathrm{~m}$ ): beam halo with $z=1 \sigma_{z}$ (top left); beam halo with $z=2 \sigma_{z}$ (top right); beam halo with $z=3 \sigma_{z}$ (bottom left); beam halo with $z=4 \sigma_{z}$ (bottom right).

\section{ACKNOWLEDGMENTS}

The authors would like to thank Dr. C. Bracco for providing a collimation script in MATLAB and help with LHC collimation simulations. The authors would also like to thank Mr. J. Barranco, Dr. R. Calaga, Dr. U. Dorda, Dr. M. Giovannozzi, Dr. J.-P. Koutchouk, Dr. F. Schmidt, Dr. T. Weiler, and Mr. S. White for helpful discussions and their comments. This work was supported by the European Community-Research Infrastructure Activity under the FP6 "Structuring the European Research Area" program (CARE, Contract No. RII3-CT-2003-506395), and under the FP7 "Capacities Specific Programme" (EuCARD, under Grant Agreement No. 227579).

[1] O. Brüning et al., CERN Report No. 2004-003, 2004.

[2] R. Palmer, SLAC Report No. SLAC-PUB-4707, 1988.

[3] K. Oide and K. Yokoya, Phys. Rev. A 40, 315 (1989).

[4] J. Irwin, Nucl. Instrum. Methods Phys. Res., Sect. A 298, 460 (1990).
[5] F. Zimmermann and U. Dorda, in Proceedings of LHCLUMI-05 Workshop, Arcidosso, Italy (Report No. CAREConf-06-014-HHH, 2005).

[6] K. Ohmi, K. Hirata, and K. Oide, Phys. Rev. E 49, 751 (1994); E. Forest, Phys. Rev. E 58, 2481 (1998).

[7] J. Rossbach and P. Schmüser, CERN Report No. 94-01, Vol. I, 1994, p. 69.

[8] Y.-P. Sun, F. Zimmermann, R. Tomás, R. Assmann, J. Barranco, T. Weiler, R. Calaga, and A. Morita, Phys. Rev. ST Accel. Beams 12, 101002 (2009).

[9] O. Brüning, R. de Maria, and R. Ostojic, LHC Project Report No. 1008, 2007.

[10] Y.-P. Sun, R. Tomás, and F. Zimmermann, Report No. LHC-AB-Note-033, 2008.

[11] C. Bracco, Ph.D. thesis, Ecole Polytechnique Lausanne, Lausanne, Switzerland [Report No. CERN-THESIS-2009031, 2009.

[12] R. Assmann, LHC Project Note No. 277, 2002.

[13] R. Assmann et al., in Proceedings of the 10th European Particle Accelerator Conference, Edinburgh, Scotland, 2006 (EPS-AG, Edinburgh, Scotland, 2006), p. 986; see also http://lhc-collimation-project.web.cern.ch/lhccollimation-project/.

[14] F. Schmidt, CERN Report No. SL/94-56 (AP), 1994. 